\title{
Microarray analysis of primary epithelial and fibroblast cells in chronic rhinosinusitis without nasal polyps*
}

\author{
Stephen L. Ball1,2, Simon J. Cockell ${ }^{3}$, Janet A. Wilson ${ }^{2,4}$, Derek A. Mann', \\ Andrew J. Fisher ${ }^{1}$ \\ ' Fibrosis Research Group, Institute of Cellular Medicine, Newcastle University, United Kingdom \\ 2 Freeman Hospital Department of Otolaryngology Head and Neck Surgery, Newcastle upon Tyne, United Kingdom \\ ${ }^{3}$ Bioinformatics Support Unit, Faculty of Medical Sciences, Newcastle University, United Kingdom
}

Rhinology 58: 6, $581-587,2020$

https://doi.org/10.4193/Rhin19.319

*Received for publication:

September 15, 2019

Accepted: April 29, 2020

\begin{abstract}
Background: Chronic rhinosinusitis is an inflammatory condition with an as yet unknown pathophysiology. We aimed to detect clusters of differentially regulated genes in the epithelial and fibroblast cells of patients with Chronic Rhinosinusitis without nasal polyposis (CRSsNP) and healthy controls.
\end{abstract}

Methodology: Carefully phenotyped CRSsNP and healthy control participants were recruited. Primary cultures of isolated epithelial and fibroblast cells were established. Whole transcriptome analysis of the cells was performed using microarrays and replicated with quantitative RT-PCR and immunohistochemistry.

Results: Fibroblast cells from CRSsNP patients showed a significant upregulation ( $>2 \mathrm{x}$ ) of the transcription factor NFE2L3 when compared to healthy controls by microarray with multiple hypothesis testing correction, qRT-PCR and immunohistochemistry.

Conclusions: Here we have utilized microarray analysis to search for differentially expressed genes in isolated patient derived epithelial and fibroblast cells. The transcription factor NFE2L3 has been shown to be upregulated in fibroblast cells consistent with increasing evidence that fibroblasts play a key role in tissue specific inflammation within the paranasal sinuses.

Key words: Rhinosinusitis, fibroblast, epithelial, microarray, NFE2L3

\section{Introduction}

Chronic rhinosinusitis (CRS) has an estimated European prevalence of $11 \%^{(1)}$, with concomitant cost and morbidity ${ }^{(2,3)}$. The precise pathophysiology of this common condition remains, however, elusive. It is diagnosed by a combination of characteristic symptoms, endoscopic and radiological findings and is currently phenotyped as being with (CRSwNP) or without (CRSsNP) nasal polyposis. This broad classification is being further investigated with emerging endotypes, or sub-phenotypes to more precisely classify these two heterogeneous groups ${ }^{(4)}$. The underlying histological appearances are well characterised, though at present do not form part of the current diagnostic criteria ${ }^{(5)}$. All subtypes of CRS see an expansion and recruitment in terms of immune and inflammatory cells $s^{(6)}$, though the precise factors that regu- late such a chemotaxis and proliferation are under investigation. As yet no study has used microarrays to analyse the transcriptome of nasal epithelial and fibroblast cells in CRSsNP.

Emerging evidence from other chronic inflammatory conditions highlights the role of stromal cells such as fibroblasts in orchestrating and perpetuating tissue specific inflammatory responses ${ }^{(7-10)}$. Recent work has highlighted the potential for sinonasal stromal cells including fibroblasts to contribute to the inflammatory environment of the upper airways $s^{(11-13)}$. Histological studies demonstrate an increase in fibrosis to be particularly important in CRSsNP. The sinonasal epithelium is the first point of contact between the external world and the respiratory system and therefore must play a critical role in sensing and 
discriminating stimuli that are potentially harmful from the many harmless particles that make up our environment. To do this the sinonasal tract has a variety of receptors, of which the Toll-like pathogen pattern recognition receptors are possibly the best characterised ${ }^{(14)}$. Sinonasal epithelium has been shown to express all the known human Toll-like receptors with to date no documented differences in expression or downstream signaling between healthy and diseased individuals, with perhaps the exception of Toll-like receptor 9 in nasal polyposis ${ }^{(15)}$. Nonetheless, the characteristic morphology of inflammatory sinonasal disease implies activation of cellular and molecular mechanisms. We hypothesized that concomitant changes should be detectable in the cellular nucleic acid transcriptome - i.e. the RNA transcripts in the cell of genes being actively expressed. Differentially expressed genes in turn may present possible novel CRS therapeutic targets. In particular, we hypothesized that nasal fibroblasts may have a role in driving and maintaining the sinonasal inflammatory processes seen in chronic rhinosinusitis.

We aimed to investigate the role of nasal epithelial and fibroblast cells in the pathophysiology of CRSsNP by comparing the transcriptome of the component CRSsNP nasal epithelial and fibroblast cells with those in health, using DNA microarray clusters of differentially expressed genes. We isolated the nasal epithelial and fibroblast cells from patients with chronic sinusitis without nasal polyposis, and thereby removed all the recruited immune cells from the tissues being analysed.

\section{Materials and methods}

\section{Participant recruitment}

Carefully phenotyped non-allergic CRSsNP patients $(n=12)$ undergoing elective operations for CRS according to the EPOS 2012 international consensus criteria ${ }^{(16)}$ were recruited with written informed consent following ethical approval by the National Health Service National Research Ethics Service Committee North East (REC reference 13/NE/0099). Control participants undergoing elective operations that use the nose as an access route for skull base procedures in the absence of sinusitis symptoms, endoscopic or radiological sinusitis findings were recruited as a comparator group $(n=12)$. Participants were nonallergic based on their clinical history, were invited for definitive skin prick testing and free of corticosteroids via all routes for the preceding two weeks. Lund-Mackay CT scan and SNOT-22 scores were recorded for each participant. The seven rhinological items of the SNOT-22 score were summated to give the rhinological symptom specific SNOT-22 score (analagous to Browne et al. ${ }^{(17)}$ with SNOT-20). The seven items are scored zero to five, and areneed to blow nose, sneezing, runny nose, post nasal discharge, thick nasal discharge, facial pain/pressure and blockage/congestion. The rhinological symptoms component SNOT-22 score ranges from 0 to 35 .
Isolation of samples and cell culture

All recruited participants had a microbiological swab taken from their middle meatus for culture, a cytological brushing for the generation of epithelial cells and a tissue biopsy for (i) the generation of fibroblast cells and (ii) tissue sections for histological analysis. Epithelial cells were grown from the brushings to near confluence at passage zero (P0), without trypsinisation or splitting of the cells. The cells were grown in submerged tissue culture conditions with Lonza BEGM media supplemented with $100 \mathrm{iu} / \mathrm{ml}$ penicillin/streptomycin. Fibroblast cells were cultured using an outgrowth technique from tissue biopsies to P1 in Sigma DMEM 5671supplemented with $100 \mathrm{iu} / \mathrm{ml}$ penicillin/ streptomycin, $10 \%$ fetal calf serum, $2 \mathrm{mM} \mathrm{L-Glutamine} \mathrm{and} 5 \mathrm{ml}$ Amphotericin B. All cells were cultured in standard sterile tissue culture conditions. Cultured cells were characterized and confirmed to be epithelial or fibroblast by immunocytochemistry using a panel of epithelial and mesenchymal markers ${ }^{(18)}$.

\section{RNA extraction and quality control}

Total RNA was extracted from $1 \times 10^{6}$ cells of each sample using a Machery Nagel NucleoSpin RNA extraction kit as per manufacturer's instructions. Determination of the RNA yield and purity was performed on a nanodrop spectrophotometer and the quality checked with an Agilent 2100 Expert Bioanalyser. All RNA integrity number (RIN) scores were $>8 / 10$.

\section{Microarray procedure}

Microarray experiments were performed using the Illumina Bead Array HT12v4 to screen 47,231 gene probes per sample. The Illumina TotalPrep-96 RNA Amplification Kit was used to generate Biotin labeled (biotin-16-UTP), amplified cRNA starting from 200ng total RNA. $50 \mathrm{ng}$ of the obtained biotinylated cRNA samples was hybridized onto the Illumina HumanHT-12 v4 as per manufacturer's instructions. The samples were scanned using the Illumina iScan array scanner. There were no deviations from the Illumina protocol. RNA labeling, amplification, and hybridization were performed by The Genome Centre at Barts and The London School of Medicine and Dentistry.

\section{Microarray data analysis}

The Illumina Human HT12v4 Expression BeadChip data was background corrected in Illumina Beadstudio. Subsequent analysis proceeded using the lumi and limma packages in R (Bioconductor) ${ }^{(19-21)}$. Variant Stabilisation Transformation and Robust Spline Normalisation were applied in lumi. Only probes with a detection $p$-value $<0.01$ in at least one sample were considered valid for downstream analysis. Differential expression was detected using linear models and empirical Bayes statistics in limma. A list of genes for each comparison was generated using a Benjamini Hochberg false discovery rate corrected $p$-value of 0.05 and a fold change of 1.5 as cut-offs ${ }^{(22)}$. The raw data from the 


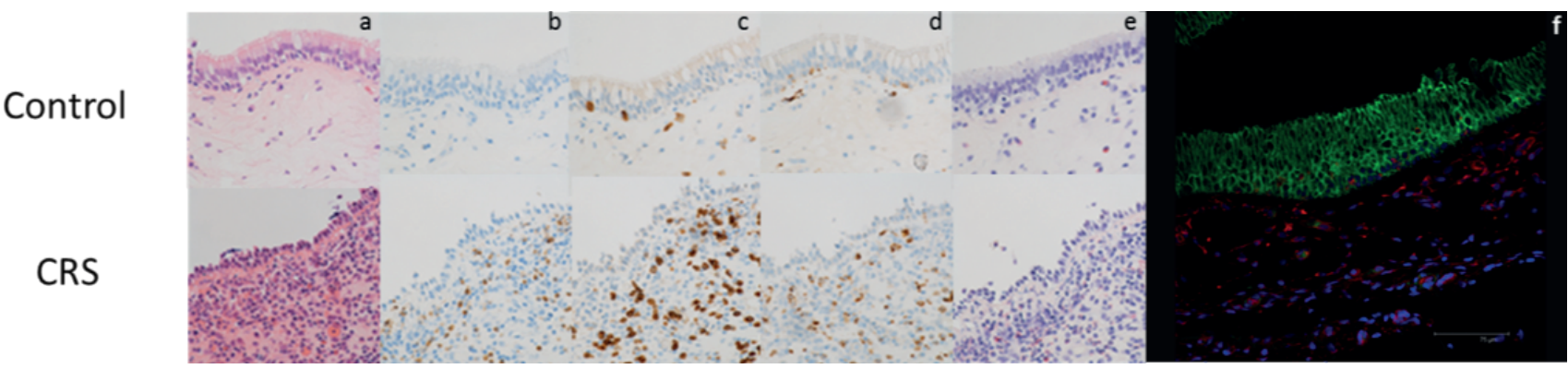

Figure 1. Histological analysis of typical recruited CRSsNP patients and healthy controls. The control panel shows the normal healthy sinonasal pseudostratified epithelium. The CRS panel demonstrates typical CRSsNP histological findings of epithelial cell loss, infiltration of immune cells in the fibroblast rich lamina propria and basement membrane thickening (a) H\&E stained tissue section. Immunohistochemical staining for (b) neutrophils (NP57 neutrophil elastase antibody), (c) T-cells (CD3), (d) macrophages (CD68), (e) eosinophils (Sirius red), (f) - Immunohistochemical staining for epithelial cell marker E-cadherin-FITC (green), fibroblast marker vimentin-TRITC (red) and DAPI nuclear stain (blue) to aid localisation of inflammatory cells. Magnification x40.

array has been deposited in the National Centre for Biotechnology Information Gene Expression Omnibus ${ }^{(23)}$ (NCBI GEO) public functional genomics data repository supporting Minimum Information About a Microarray Experiment (MIAME)-compliant data submissions ${ }^{(24)}$ (reference GSE69093).

\section{Quantitative real time RT-PCR}

Quantitative RT-PCR was used to replicate the findings of the microarray using the standard $\Delta \Delta \mathrm{Ct}$ (cycle threshold) method; $\Delta \Delta \mathrm{Ct}=2 \wedge(\mathrm{Ct}$ of reference gene $-\mathrm{Ct}$ of candidate gene). CDNA was prepared from isolated RNA samples using the BIORAD iScript CDNA synthesis kit as per manufacturer's instructions. 10ng of CDNA template was used per qRT-PCR reaction using SYBR Green JumpStart Taq Ready Mix on an Applied Biosystems 7500 Real-Time PCR System for 35 cycles. $1 \mu$ l of forward and reverse primers (NFE2L3 - as identified from the microarray analysis, forward TCCCAGCATGAGGAAAATGA, reverse TTCTGCCTCCCAGTCAGGTTT $^{(25)}$ ) were supplied by Eurofins per reaction. Expression levels of mRNA were compared to those of the healthy controls for relative mRNA expression levels. Unpaired t-tests were used to analyse the difference in mRNA expression between healthy controls and CRS patients. Products formed in the qRT-PCR reactions were verified by $2 \%$ agarose gel electrophoresis and compared to a 100 base pair ladder.

\section{Immunohistochemical staining}

Formalin fixed paraffin embedded tissue sections were produced from tissue biopsy samples. Sections were de-waxed and rehydrated twice in Clearene followed by $100 \%$ and $70 \%$ ethanol each for 5 minutes. Antigen retrieval was performed in $1 \mathrm{mM}$ EDTA at pH8 in a microwave at 700 watts for 15 minutes. Nonspecific binding was blocked using $5 \%$ bovine serum albumen in PBS with $0.2 \%$ Tween 20 (5\% BSA PBST) at room temperature for 1 hour. To replicate the findings of the micro array and RT-
PCR, anti NFE2L3 primary antibody (LSBio LS-B8066) at 1:200 dilution was incubated overnight at $4{ }^{\circ} \mathrm{C}$ in $5 \%$ BSA PBST. TRITC conjugated secondary antibody (Sigma T6778) at 1:100 dilution was incubated in 5\% BSA PBST at room temperature and in darkness. Sections were counterstained and mounted with vectashield DAPI containing mounting medium (Vector laboratories $\mathrm{H}$-1200). Slides were imaged on a Nikon A1 on a Nikon Eclipse NI-E upright stand with a x20 0.75Na Plan Apo lens running Nikon elements 4.30.02. Quantification of images was performed using Image J with readings for the epithelial fluorescence (TRITC red). The values were expressed as corrected integrated density, with removal of any background fluorescence.

\section{Results}

Clinical data

Recruited participants showed significant differences in their rhinological SNOT-22 symptom and Lund Mackay CT scan scores between control $(n=12)$ and CRSsNP $(n=12)$ patients $(p<0.0001)$. There were no significant age or sex differences. Microbiological analysis of middle meatal swabs from all participants identified only a growth of Staphylococcus aureus, present in two CRSsNP participants. Tissue biopsies analysed for histological evidence of either healthy sinonasal tissue or CRSsNP are shown in Figure 1 as previously described by Kou et al. ${ }^{(26)}$ and Fokkens et al. ${ }^{(27)}$. The tissue biopsies demonstrate typical examples of the histological characterization, immune cell infiltration and distribution in both healthy controls and CRSsNP patients. Control participants showed the normal healthy sinonasal pseudostratified epithelium. CRSsNP participants demonstrated confirmatory pathological findings of epithelial cell loss, infiltration of immune cells in the fibroblast rich lamina propria and basement membrane thickening. Isolated cells were confirmed epithelial and fibroblast respectively with epithelial and fibroblast markers (Supplemental Figure 1). Assessment with transmission electron 


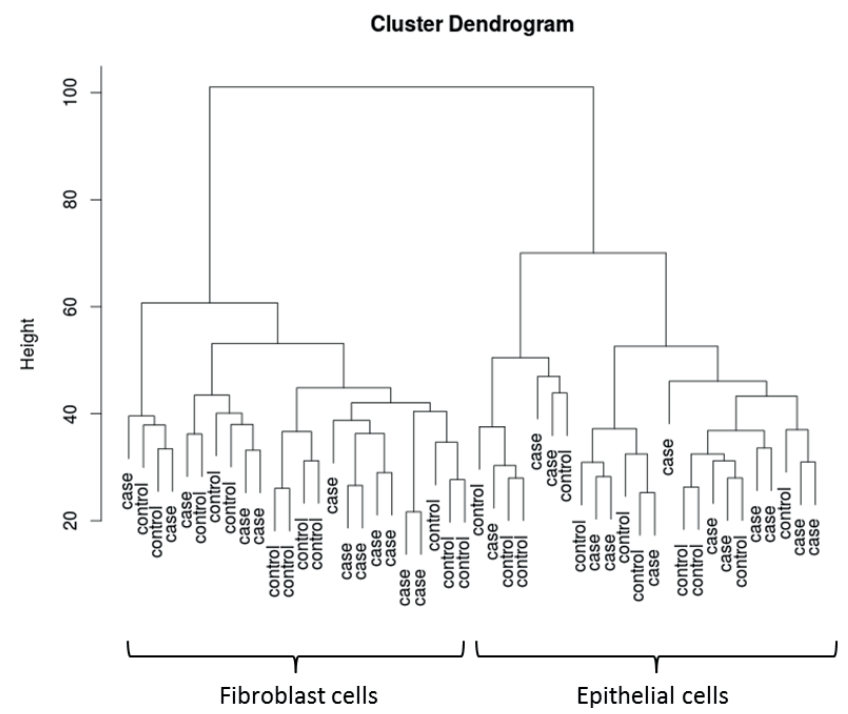

Figure 2. Dendrogram produced by hierarchical clustering of CRS and healthy control gene samples. Each gene starts in its own cluster and the most similar genes according to the Euclidian distance similarity metric are merged. The similarity metrics are recalculated between the genes and the new cluster and the process is repeated until all genes are in a single cluster. The samples are principally separated on the basis of the cell type of origin, either epithelial or fibroblast rather than case (CRSsNP) or control.

microscopy excluded a diagnosis of primary ciliary dyskinesia base on the typical ' $9+2$ ' axoneme.

\section{Microarray data}

The initial comprehensive microarray analysis was a hierarchical clustering to explore for differences in gene expressions between healthy controls ( $n=12)$ and CRSsNP patients $(n=12)$. Each gene starts in its own cluster and the most similar genes are merged according to the Euclidian distance similarity metric. The similarity metrics are recalculated between the genes and the new cluster and the process is repeated until all genes are in a single cluster. Hierarchical clustering analysis clearly separated our samples based on their cell type, confirming the difference between epithelial and fibroblast samples (Figure 2). The cluster analyses, however, did not significantly discriminate between healthy controls and CRSsNP participants.

Microarray analysis of isolated primary nasal epithelial cells was performed following Benjamini Hochberg correction for multiple hypothesis testing. No significantly differential (>50\% up or down) gene expression was found.

A similar comparison of fibroblast cells from control and CRSsNP participants identified one significantly differentially expressed gene (Figure 3), nuclear factor erythroid-derived 2-like 3

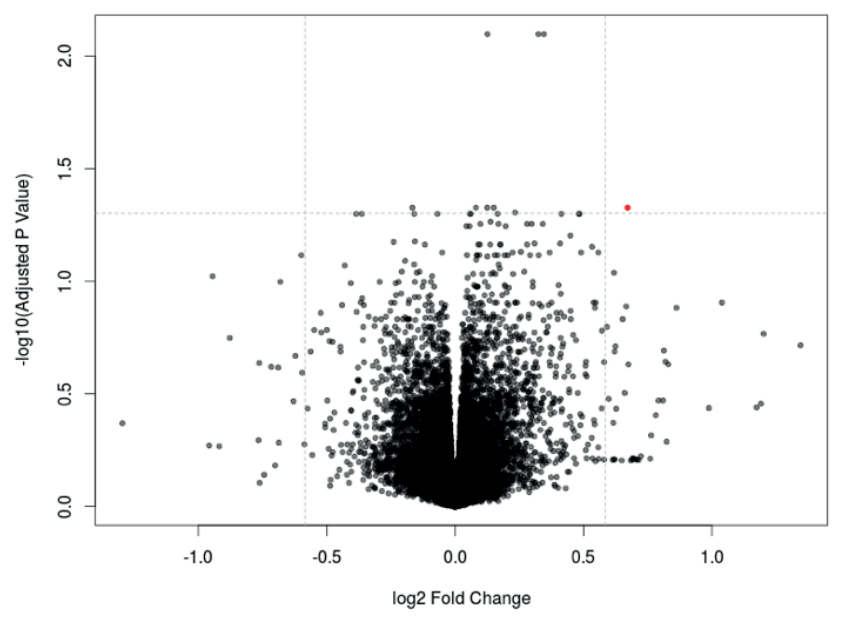

Figure 3. Isolated fibroblast cells' volcano plot to identify any statistically significant differential gene expression between CRS and control samples following Benjamini-Hochberg multiple hypothesis testing correction. Points outside the dashed vertical lines demonstrate either a $50 \%$ up or down regulation in gene expression. Points above the dashed horizontal line show a statistically significant difference of greater than $p<0.05$. Points that satisfy both differential expression criteria and statistical significance have been coloured red. In this instance one gene fulfils both criteria; NFE2L3. (a)

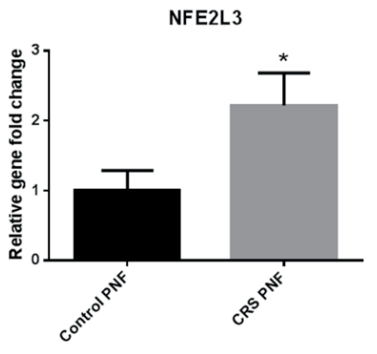

(b)

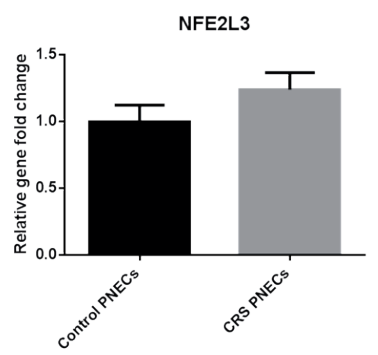

Figure 4. qRT-PCR replication of NFE2L3 gene expression between CRSsNP ( $n=12)$ and control fibroblasts (PNFs, $n=12)$ and epithelial cells (PNECs, $n=12$ ). Relative gene expression has been normalized to healthy control cells. Figure 4(a) shows a statistically significant $>2$-fold upregulation in the NFE2L3 gene in CRSsNP fibroblast cells, ${ }^{*}=p<0.05$ (0.0352). Figure 4(b) demonstrates an increase in NFE2L3 in CRSsNP epithelial cells compared to control cells, however this does not reach statistical significance, $p=0.1980$.

(NFE2L3, $p=0.000015, p=0.0471$ following multiple hypothesis testing correction). NFE2L3 is a transcription factor with potential roles in inflammation that was $60 \%$ upregulated in CRSsNP fibroblast cells compared to healthy controls.

qRT-PCR and immunohistochemical replication Quantitative real time RT-PCR was used to replicate the findings 


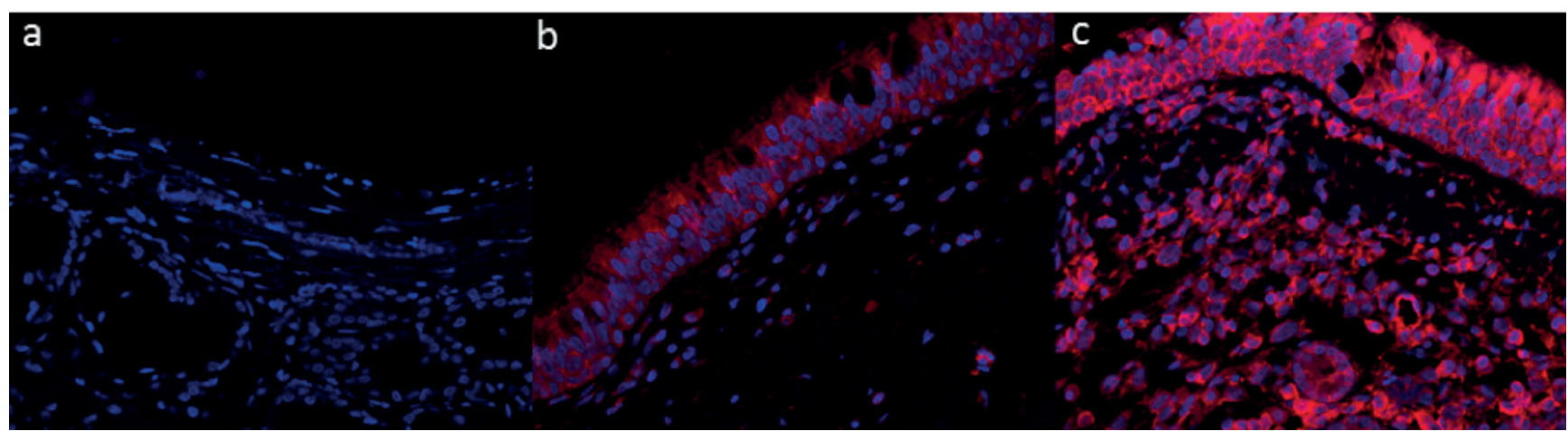

Figure 5. Fluorescent immunohistochemical staining of CRSsNP and control tissue sections. Tissues have been stained with anti NFE2L3 primary antibody and TRITC conjugated secondary antibody (red). Sections have been counterstained with DAPI nuclear staining (blue). (a) no primary control section (b) healthy control sample (c) CRS tissue sample. Magnification x40. Corrected integrated density to remove background fluorescence shows the epithelium in section (b) to have a value of 37.6 and (c) 80.7, the subepithelial stromal tissue in (b) has a value of 4.5 and (c) 102.9 .

of the microarray in control $(n=12)$ and CRSsNP samples $(n=12)$ with 15 primer pairs of the most differentially expressed microarray probes including NFE2L3. qRT-PCR reactions replicated and confirmed the findings of the microarray, with the transcription factor NFE2L3 showing a two-fold increase in expression in fibroblasts derived from CRSsNP patients compared to controls, $p=0.0352$ (Figure 4a). Similar to the microarray, the remainder of qRT-PCR primer pairs did not reveal any statistically significant expression of mRNA between control and CRSsNP epithelial and fibroblast samples using unpaired t-tests. Fluorescent immunohistochemical staining of healthy control and CRSsNP tissue sections was used in addition to determine if there was any difference in the amounts of the NFE2L3 transcription factor protein within the tissues. Figure 5 shows typical examples of the expression of NFE2L3 in healthy control and CRSsNP tissue sections. Quantitatively, there is an increase in staining highlighted by the increased signal from CRSsNP samples. The difference is most marked in the sub epithelial tissues, corrected integrated density to remove any background fluorescence in the healthy control epithelium in Figure (5b) has a value of 37.6 and the CRSsNP (Figure 5c) sample has a corrected integrated density value for fluorescence of 80.7. The subepithelial stromal tissues show an up-regulation in their integrated density from 4.5 in healthy control (Figure 5b) to 102.9 in CRSsNP (Figure 5c).

\section{Discussion}

In this study we have recruited a cohort of carefully phenotyped CRSsNP patients and healthy controls and isolated their component structural sinonasal epithelial and fibroblast cells in tissue culture conditions to P0 \& P1. Using these isolated epithelial and fibroblast cells as a starting template, we have performed a genome wide microarray to look for differentially expressed genes as potential candidates for further mechanistic investigation and therapeutic targets. Our bioinformatics analysis of the microarray data demonstrates that fibroblast cells from CRS patients show higher expression of the transcription factor NFE2L3 than do fibroblasts from control participants. Somewhat surprisingly, there were no statistically significantly differentially expressed genes between CRS \& control epithelial cells. Quantitative real time RT-PCR replication with a series of candidate genes has confirmed the findings of our microarray analysis. Immunohistochemical staining of tissue biopsies for NFE2L3 protein has provided further confirmation of the micro array and RT-PCR findings. Quantification of the immunohistochemical staining shows the most significant increase in NFE2L3 protein within the fibroblast rich sub-epithelial layer with a corrected integrated density that is 23 times higher in CRSsNP tissue than in healthy control tissue. As in the PCR data there is an increase in the NFE2L3 within the epithelial layer, though this is not as dramatic. Nuclear factor erythroid-derived 2-like 3 (NFE2L3) belongs to the highly evolutionarily conserved Cap'n'Collar (CNC) protein subgroup of basic region-leucine transcription factors ${ }^{(28)}$. It contains a 43 amino acid CNC domain specific to its DNA binding activity $^{(29)}$. There is established evidence for a role of NFE2L3 in inflammation in both in vitro and in vivo and via human genome wide association studies. NFE2L3 is a member of a family of genes that act as negative regulators of a collection of defensive genes in response to oxidative stress ${ }^{(30-32)}$. Both NFE2L3 protein and mRNA are upregulated in tissue culture by tumour necrosis factor and interferon- $\gamma^{(33,34)}$, which are key Th1 cytokines in the pathophysiology of CRSSNP(35). NFE2L3 has been shown to be an important factor in murine models of oxidative lung injury ${ }^{(36,37)}$. Genome wide association studies have further identified NFE2L3 to be associated with endometriosis ${ }^{(38)}$, obesity and diabetes ${ }^{(39)}$. It is therefore plausible that the transcription factor NFE2L3 may have a role to play in the complex chronic inflammation seen within the sinonasal cavity.

In contrast, despite the upregulation of NFE2L3 within sinonasal fibroblasts it is perhaps surprising that more differentially 
expressed genes were not identified between CRSsNP patients and healthy controls from primary cultures of their sinonasal epithelial and fibroblast cells. Although the multiple hypothesis testing correction is strict, over forty seven thousand different human probes were screened for each sample. The paradox may arise from patient selection and inter individual variation. However, as illustrated by the clinical, radiological and histological data, all patients were carefully phenotyped for CRSsNP. All participants were non-allergic based on their clinical history and were invited for definitive skin prick testing and free of corticosteroids via all routes for the preceding two weeks. The sample size in this exploratory study is relatively small in part due to the technical requirements and cost of the microarrays. The lack of differentially expressed genes could in part be due to minor differences in medications or smoking status of participants, though with respect to these variables there were no major differences between each patient group. The lack of differentially expressed genes may also be related to the fact that the samples studied were quiescent cells grown in sterile tissue culture conditions, removed from the body and the complex environmental stimuli of the sinonasal cavity. All cells were grown in tissue culture conditions, a sterile environment together with growth factors and antibiotics to aid the successful proliferation of the primary human sinonasal cell lines established. Both cell types used early passage cells of either PO for epithelial cells and P1 for fibroblast cells rather than those that have undergone multiple cell divisions and trypsinisations in culture. Within the cell culture, however, the presence of growth factors and antibiotics may have two-fold effects. Firstly, the sterile media with supplemental antibiotics removes the normal microenvironment of the sinonasal cavity, be it planktonic bacteria, viral $^{(40)}$ or biofilm ${ }^{(41)}$ stimulation. Secondly the growth medium supplements to promote successful proliferation of primary cells in culture may provide supra-physiological stimulus for growth and cellular activity that actually disguises any difference in gene expression between the healthy control and CRSsNP cells. Along with the growth factor supplements and corticosteroids in the media which may suppress inflammation and hence RNA expression it must also be mentioned that the epithelial cells are grown in submerged culture rather than at an air liquid (ALI) interface. Air liquid interface culture perhaps more accurately re- presents the mucosal environment in the sinonasal cavities and may have an impact on cellular function and gene transcription. It is also worth commenting that a lack of major differences at the gene and RNA level does not always equate to a lack of difference at the functional level of the gene product i.e. the proteins translated from the individual genes and RNAs. To investigate the low number of gene targets identified here by microarray further it would certainly be interesting to see how the presented microarray of primary cellular samples compares to either a transcriptome analysis of the parent tissues that the cells were derived from. Secondly, it would be interesting to investigate the responses and downstream signaling following primary cell stimulation with disease relevant stimuli found in the sinonasal microenvironment.

\section{Conclusion}

In conclusion, we have identified increased expression of the transcription factor NFE2L3 by microarray, quantitative RT-PCR and fluorescent immunohistochemistry in isolated primary nasal fibroblast cells from a carefully phenotyped cohort of CRSsNP and healthy control patients. This finding adds further evidence to the potential emerging role of the fibroblast in inflammatory upper airway conditions.

\section{Acknowledgements}

We wish to acknowledge the help and contribution of our patients in the preparation and completion of this work. The work has been funded by a Wellcome Trust Clinical Research Training Fellowship: Grant number: 100201/Z/12/A provided to Dr Stephen Ball.

\section{Authorship contribution}

The work presented has been carried out and the manuscript written by SB. Bioinformatics analysis was performed by SB with the support of SC. AF, DM \& JW contributed to the conception and design of the study, supervised SB's doctoral thesis and coauthored the manuscript.

\section{Conflict of interest}

The authors have no conflict of interest to declare.

\section{References}

1. Hastan D, Fokkens WJ, Bachert C, et al. Chronic rhinosinusitis in Europe--an underestimated disease. A GA(2)LEN study. Allergy. 2011;66(9):1216-1223.

2. Ray NF, Baraniuk JN, Thamer M, et al. Healthcare expenditures for sinusitis in 1996: contributions of asthma, rhinitis, and other airway disorders. J Allergy Clin Immunol. 1999;103(3 Pt 1):408-414.

3. Smith KA, Orlandi RR, Rudmik L. Cost of adult chronic rhinosinusitis: A systematic review. Laryngoscope. 2015;125(7):15471556.

4. Akdis CA, Bachert C, Cingi C, et al. Endotypes and phenotypes of chronic rhinosinusitis: a PRACTALL document of the European Academy of Allergy and Clinical Immunology and the American Academy of Allergy, Asthma \& Immunology. J Allergy Clin Immunol.. 2013;131(6):1479-1490.

5. Fokkens WJ, Lund VJ, Mullol J et al. EPOS
2012: European position paper on rhinosinusitis and nasal polyps 2012. A summary for otorhinolaryngologists. Rhinology. 2012;50(1):1-12.

6. Bachert C, Zhang N, van Zele T, Gevaert P. Chronic rhinosinusitis: from one disease to different phenotypes. Pediatr Allergy Immunol. 2012;23 Suppl 22:2-4.

7. Naylor AJ, Filer A, Buckley CD. The role of stromal cells in the persistence of chronic inflammation. Clin Exp Immunol. 
2013;171(1):30-35.

8. Suwara MI, Green NJ, Borthwick LA et al. IL-1alpha released from damaged epithelial cells is sufficient and essential to trigger inflammatory responses in human lung fibroblasts. Mucosal immunol. 2014;7(3):684-693.

9. Alkhouri H, Poppinga WJ, Tania NP, Ammit A, Schuliga M. Regulation of pulmonary inflammation by mesenchymal cells. Pulm Pharmacol Ther. 2014;29(2):156-165

10. Patel R, Filer A, Barone F, Buckley CD. Stroma: fertile soil for inflammation. Best Pract Res Clin Rheumatol. 2014;28(4):565576.

11. Early SB, Hise K, Han JK, Borish L, Steinke $J W$. Hypoxia stimulates inflammatory and fibrotic responses from nasal-polyp derived fibroblasts. Laryngoscope. 2007;117(3):511515.

12. Rudack C, Hermann W, Eble J, Schroeder JM Neutrophil chemokines in cultured nasa fibroblasts. Allergy. 2002;57(12):1159-1164.

13. Ball SL, Mann DA, Wilson JA, Fisher AJ. The Role of the Fibroblast in Inflammatory Upper Airway Conditions. Am J Pathol. 2016;186(2):225-233.

14. Ooi EH, Wormald PJ, Tan LW. Innate immunity in the paranasal sinuses: a review of nasal host defenses. Am J Rhinol. 2008;22(1):1319

15. Zhao CY, Wang X, Liu M, Jin DJ. Microarray gene analysis of Toll-like receptor signaling elements in chronic rhinosinusitis with nasal polyps. Int Arch Allerg Immunol. 2011;156(3):297-304.

16. Fokkens WJ, Lund VJ, Mullol J et al. European Position Paper on Rhinosinusitis and Nasal Polyps 2012. Rhinol Suppl. 2012(23):3 p preceding table of contents, $1-298$.

17. Browne JP, Hopkins C, Slack R, Cano SJ. The Sino-Nasal Outcome Test (SNOT): can we make it more clinically meaningful? Otolaryngol Head Neck Surg 2007;136(5):736-741.

18. Ball SL, Suwara MI, Borthwick LA, Wilson JA, Mann DA, Fisher AJ. How reliable are sinonasal cell lines for studying the pathophysiology of chronic rhinosinusitis? Ann Otol Rhinol Laryngol. 2015;124(6):437-442.

19. Du P, Kibbe WA, Lin SM. Iumi: a pipeline for processing Illumina microarray. Bioinformatics. 2008;24(13):1547-1548

20. Gentleman RC, Carey VJ, Bates DM et al. Bioconductor: open software development for computational biology and bioinformatics. Genome Biol. 2004;5(10):R80.
21. Smyth GK. Limma: linear models for microarray data. Bioinformatics and Computational Biology Solutions using $\mathrm{R}$ and Bioconductor. : Gentleman RC CV, Dudoit S, Irizarry R, Huber W, editor. New York: Springer; 2005.

22. Benjamini $Y$, Hochberg Y. Controlling the False Discovery Rate: A Practical and Powerful Approach to Multiple Testing. J Royal Stat Soc. 1995;57(1):289-300.

23. NCBI. Gene Expression Omnibus 2015 [GEO is a public functional genomics data repository supporting MIAME-compliant data submissions. Array- and sequence-based data are accepted. Tools are provided to help users query and download experiments and curated gene expression profiles. ]. Available from: http://www.ncbi.nlm. nih.gov/geo/.

24. Brazma A, Hingamp P, Quackenbush J et al. Minimum information about a microarray experiment (MIAME)-toward standards for microarray data. Nat Genet. 2001;29(4):365371

25. Korecka JA, van Kesteren RE, Blaas E et al. Phenotypic characterization of retinoic acid differentiated SH-SY5Y cells by transcriptional profiling. PloS One. 2013;8(5):e63862.

26. Kou W, Hu GH, Yao HB et al. Regulation of transforming growth factor-betal activation and expression in the tissue remodeling involved in chronic rhinosinusitis. ORL J Otorhinolaryngol Relat Spec. 2012;74(3):172-178

27. Fokkens WJ, Lund VJ, Hopkins $C$ et al. European Position Paper on Rhinosinusitis and Nasal Polyps 2020. Rhinology. 2020;58(Suppl S29):1-464

28. Sykiotis GP, Bohmann D. Stress-activated cap'n'collar transcription factors in aging and human disease. Sci Signal. 2010:3(112):re3.

29. Toki T, Itoh J, Kitazawa J et al. Human small Maf proteins form heterodimers with CNC family transcription factors and recognize the NF-E2 motif. Oncogene. 1997;14(16):1901-1910.

30. Sankaranarayanan K, Jaiswal AK. Nrf3 negatively regulates antioxidant-response element-mediated expression and antioxidant induction of $\mathrm{NAD}(\mathrm{P}) \mathrm{H}$ :quinone oxidoreductase 1 gene. J Biol Chem. 2004;279(49):50810-50817.

31. Jaiswal AK. Regulation of antioxidant response element-dependent induction of detoxifying enzyme synthesis. Methods Enzymol. 2004;378:221-238.

32. Jaiswal AK. Regulation of genes encoding
NAD(P)H:quinone oxidoreductases. Free Radic Biol Med. 2000;29(3-4):254-262.

33. Chenais B, Derjuga A, Massrieh W et al. Functional and placental expression analysis of the human NRF3 transcription factor. Mol Endocrinol. 2005;19(1):125-137.

34. Kitaya K, Yasuo T, Yamaguchi T, Fushiki $\mathrm{S}$, Honjo $\mathrm{H}$. Genes regulated by interferon-gamma in human uterine microvascular endothelial cells. Int J Mol Med 2007;20(5):689-697.

35. Van Crombruggen K, Zhang N, Gevaert P, Tomassen P, Bachert C. Pathogenesis of chronic rhinosinusitis: inflammation. J Allergy Clin Immunol. 2011;128(4):728-732.

36. Chevillard G, Nouhi Z, Anna D, Paquet M, Blank V. Nrf3-deficient mice are not protected against acute lung and adipose tissue damages induced by butylated hydroxytoluene. FEBS Lett. 2010;584(5):923-928.

37. Paola RD, Cuzzocrea S. Peroxisome proliferator-activated receptors and acute lung injury. PPAR Res. 2007;2007:63745.

38. Painter JN, Anderson CA, Nyholt DR et al. Genome-wide association study identifies a locus at 7p15.2 associated with endometriosis. Nat Genet. 2011:43(1):51-54.

39. Heid IM, Jackson AU, Randall JC et al. Meta-analysis identifies 13 new loci associated with waist-hip ratio and reveals sexual dimorphism in the genetic basis of fat distribution. Nat Genet. 2010;42(11):949-960.

40. Hox V, Maes T, Huvenne $W$ et al. A chest physician's guide to mechanisms of sinonasal disease. Thorax. 2015;70(4):353-358.

41. Foreman A, Boase S, Psaltis A, Wormald PJ. Role of bacterial and fungal biofilms in chronic rhinosinusitis. Curr Allergy Asthma Rep. 2012;12(2):127-135.

\section{Dr Stephen Ball \\ Institute of Cellular Medicine \\ Newcastle University Medical School \\ Framlington Place \\ Newcastle upon Tyne, NE2 4HH \\ United Kingdom}

Tel: +44 - 01912225106

E-mail: s.l.ball@ncl.ac.uk 


\section{SUPPLEMENTARY DATA}

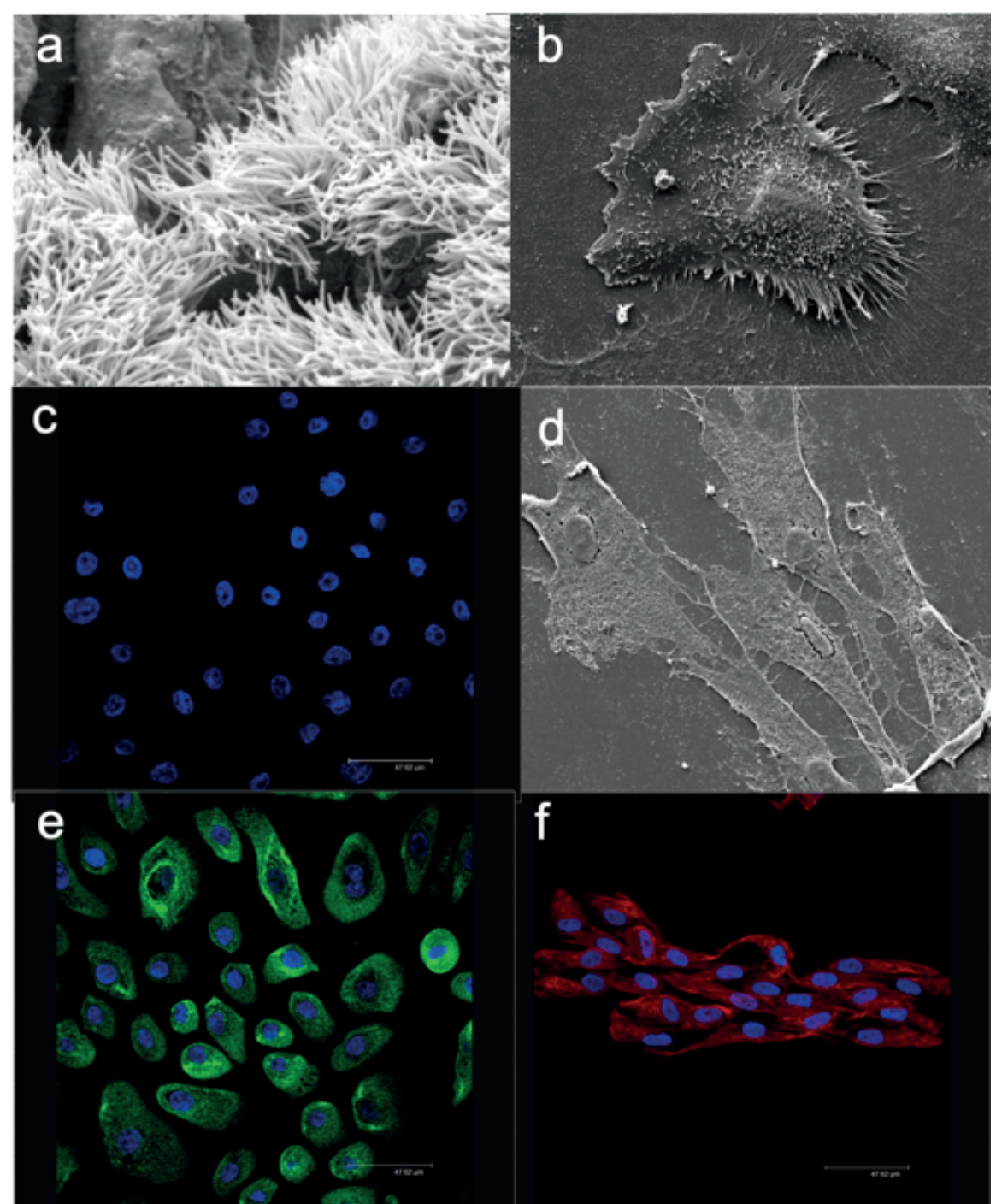

Supplementary Figure 1. Characterisation of cultured cells from patient samples (a) Scanning electron micrograph (SEM) of healthy sinonasal mucosal tissue, (b) SEM of isolated epithelial cell, (c) representative negative immunocytochemical staining for epithelial/fibroblast genes respectively, DAPI nuclear stain (blue), (d) SEM of isolated fibroblast cells, (e) epithelial cells positive staining for pan cytokeratin FITC epithelial marker (green), (f) fibroblasts staining positive for vimentin fibroblast marker (red). 